\title{
Impact of Intensive Mixing and Shearing Elements on the Effectiveness of Extrusion of Wood Polypropylene Composites
}

\author{
Karolina Głogowska ${ }^{1 *}$, Janusz W. Sikora' ${ }^{1}$ Krzysztof Ludziak' \\ 1 Department of Technology and Polymer Processing, Faculty of Mechanical Engineering, Lublin University \\ of Technology, Nadbystrzycka 36, 20-618 Lublin, Poland \\ * Correspondening author's e-mail: k.glogowska@pollub.pl
}

\begin{abstract}
The objective of this study was to determine the effect of various design solutions for intensive mixing and shearing elements located at the end of a single-screw plasticizing system on the effectiveness of wood polypropylene composite extrusion. Four types of such elements were used in the study: a pineapple mixer, a Maddock mixer, a ring mixer, and a helical mixer. For the purpose of the study, four blends were prepared containing 5, 10, 15 and $20 \%$ of wood fibre, respectively. Direct and indirect resulting factors as well as variable, constant and disturbing factors were investigated. The effectiveness of extrusion of wood polypropylene composites was determined based on results of parameters characterizing this process, such as extrudate temperature, unit consumption of energy supplied to the extruder, mass flow rate, and extrusion speed. It has been shown that the effectiveness of the extrusion process can be improved by using different mixing and shearing elements. Such screw design solutions make it possible to affect rheological phenomena occurring during melt flow in the plasticizing system, without modifying the extrusion process parameters such as screw rotation speed and polymer temperature. The literature review shows that there exist very few and general studies investigating the effect of screw design geometry, especially that of mixing and shearing elements, on the efficiency of extruding and mixing wood polypropylene materials.
\end{abstract}

Keywords: single-screw extrusion, shearing elements, mixing elements, process effectiveness, wood polypropylene composite.

\section{INTRODUCTION}

Polymer processing techniques are rapidly developing these days. The extrusion and injection molding process plays a very important role in polymer processing and are the most widely used methods for processing polymers [1-3]. There are different types of extrusion processes, such as conventional extrusion, autothermal extrusion, extrusion coating, blown film extrusion, and many others [4-6]. The extrusion method is used to manufacture everyday objects such as profiles, plates, tapes, films, containers and bottles; it also makes part of other processing methods such as bonding, calendering and granulating.

In polymer processing, phenomena occurring in the plasticizing system are of great importance for extrusion process effectiveness [7]. The plasticizing system performs several important functions, including heating, compression, mixing and conveyance [3]. Technological conditions of the extrusion process and the design of the extruder plasticizing system play a key role in polymer extrusion, too. Research on the improvement of single-screw plasticizing systems predominantly focuses on new design solutions for the barrel and screw. This is due to the fact that these elements have a crucial impact on the flow rate of a polymeric material, energy consumption of the extrusion process, and the desired quality of the extrudate. Polymer mixing is a key operation in extrusion [3]. Mixing is used to homogenize the composition and properties (predominantly frictional and mechanical) of a processed polymer, as well as to homogenize its structure [8-11]. Therefore, there exist many different screw designs. 
Screws can be made of a single piece of metal or consist of multiple pieces. On its core surface, the screw has a helix that forms a screw channel with the barrel, the channel can be continuous or discontinuous; infrequently, the screw contains intensive mixing and shearing elements, and additionally other screw channel sections [12]. In this way, it is possible to design screws of very complex geometry that provide high efficiency of polymer plasticisation [8].

To increase the efficiency of mixing plastics in the plasticizing system, different screw design solutions are employed, including screws of various shapes and sizes, provided with elements for improved mixing and shearing, known as special screws [11, 13], or/and with additional devices such as static mixers in technological extrusion lines.

A pineapple mixing section is an example of high shear elements that primarily exhibit distributive mixing characteristics. Examples of elements that are predominantly used for dispersive mixing include blister rings or grooved elements such as the Maddock [8, 10], Maillefer or Barr style mixers [3]. The mixing process can be predominantly intensified by sequence-type dividing of the streams of polymer and combining them subsequently as well as by rotating these streams. The intensive mixing and shearing elements are either located on the screw in the metering section or positioned at the end of the screw [11].

Like screws, extruder barrels may have a complex design; they may have outer grooves for cooling and their inner surface may be geometrically non-uniform. To ensure high efficiency of the extrusion process, grooved barrel zones are created at the beginning of the plasticizing system. One can distinguish passive and active grooved zones, the most widely used being the passive ones in which geometrical features of the grooves remain unchanged during the extrusion process [14]. In contrast, the geometrical features of active grooved zones, such as the inclination angle of the groove, the number of grooves, the depth of the groove, and the torsional angle of the groove, can be modified during the extrusion process without stopping the extruder [15].

There exist many different screw design solutions, most of which are patent-protected, which indicates both the complexity of the problem and the shortcomings of the screw design theory and screw design basics. The criteria for screws are complex, and it is difficult to decide on the most appropriate screw for a given application.
Due to high requirements for products made of polymeric materials, studies are conducted on improving processing methods and minimizing their imperfections in order to obtain technologically correct products characterized by high functional, aesthetic and mechanical properties.

The growing interest in wood polymer composites is evidenced, among others, by the number of producers of granulated products and end products made of wood polymer composites (WPCs). Although the most widely used WPC processing technique is extrusion $(70 \%)$, these materials can also be processed by injection moulding and press moulding. There exists a considerable body of literature on the extrusion of wood polypropylene composites, predominantly relating to mechanical and physical properties of these materials and changes in these properties due to chemical modification of the filler or the entire blend. Previous studies have also investigated water absorption, flammability and resistance to fungi of WPCs using various processing techniques [16].

The objective of the study presented in this paper is to determine the effect of using four different intensive mixing and shearing elements on the extrusion process of polypropylene filled with different contents of wood fibre.

\section{MATERIALS AND METHODS}

\section{Materials}

Eltex ${ }^{\circledR}$ P KS404N6996 polypropylene (PP) in pellet form was used in the study. Supplied by INEOS Olefins \& Polymers Europe (Rolle, Switzerland), Eltex ${ }^{\circledR}$ P KS404N6996 is a random copolymer with average ethylene content. It contains no special additives and is thus often used by manufacturers wishing to create their own formulas. Table 1 gives the basic physical and mechanical properties of this polypropylene, as specified in the technical datasheet provided by the manufacturer.

Table 1. Basic properties of the polymer used in the study, according to the technical datasheet provided by the manufacturer

\begin{tabular}{|l|c|c|}
\hline \multicolumn{1}{|c|}{ Property } & Test method & Value \\
\hline MFR $\left(230^{\circ} \mathrm{C} ; 2.16 \mathrm{~kg}\right), \mathrm{g} / 10 \mathrm{~min}$ & ISO 1133 & 7 \\
\hline Flexural Modulus, $\mathrm{MPa}$ & ISO 178 & 900 \\
\hline Tensile Stress (Yield), MPa & ISO 527-1,2 & 27 \\
\hline Melting Temperature, ${ }^{\circ} \mathrm{C}$ & ASTM & 143.5 \\
\hline
\end{tabular}




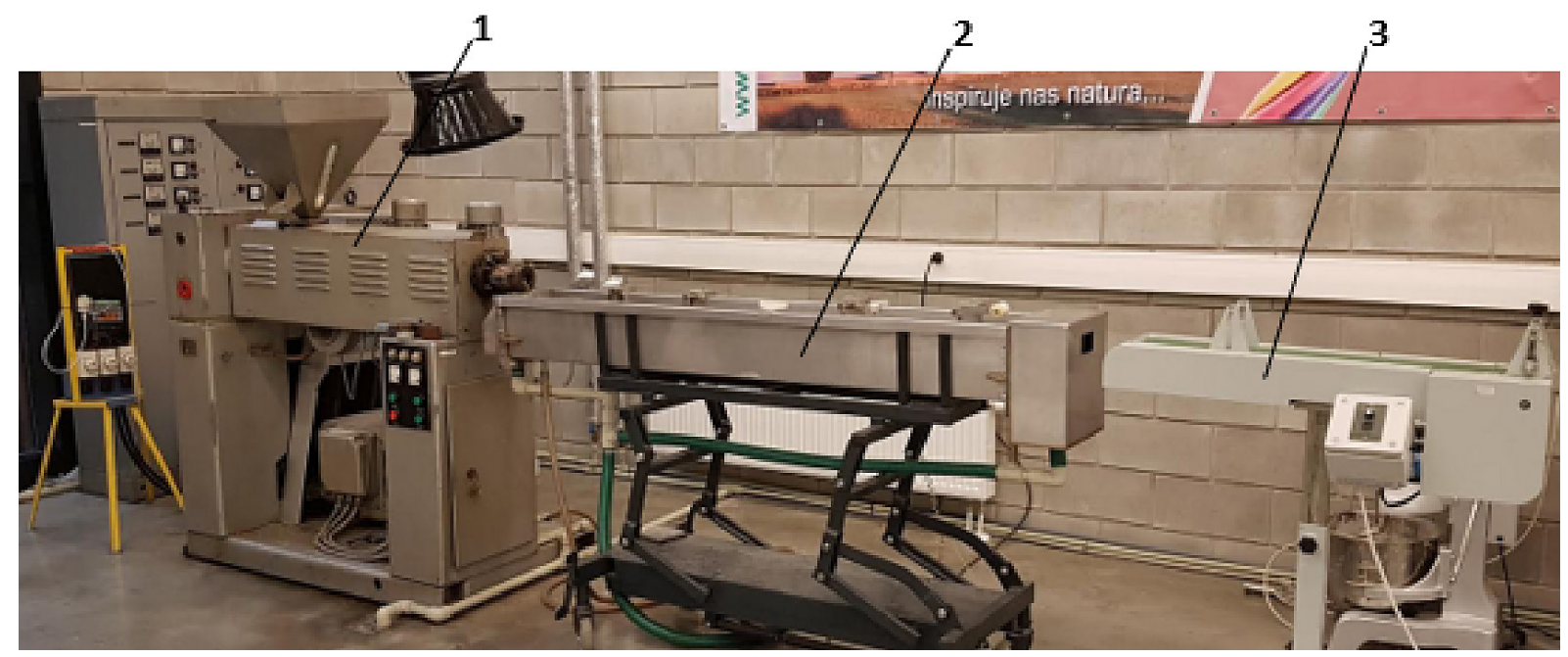

Fig. 1. Profile extrusion line: 1 - extruder, 2 - cooling tank, 3 - haul off

Ligocel C 120 wood fibre manufactured by J. Rettenmaier \& Söhne GmbH CoKG (Rosenberg, Germany) was used as filler. It is made from fibrous pinewood. Basic properties of the filler, as specified by the manufacturer, are given in Table 2 .

\section{Test Stand}

The study on the effectiveness of the extrusion process for wood polypropylene composites was carried out using a laboratory profile extrusion line, its key component being a single-screw extruder, T32-25. The laboratory extrusion line for wood polypropylene composites (Fig. 1) also consists of a cooling tank and a $100 \mathrm{~mm}$ wide and $2000 \mathrm{~mm}$ long haul off unit.

The extruder plasticizing system has four heaters, and the screw is described by an L/D ratio of 25 and an outside diameter D of $32 \mathrm{~mm}$. Different mixing and shearing elements can be mounted on the screw, each described by an L/D ratio of 4.4. Four types of intensive mixing and shearing elements were used in the experiments: a pineapple mixer (Fig. 2a), a Maddock mixer (Fig. 2b), a cut ring mixer (Fig. 2c) and a helical mixer (Fig. 2d). The rotation speed range of the extruder screw is

Table 2. Basic properties of the filler used in the study, according to the technical datasheet provided by the manufacturer

\begin{tabular}{|c|c|}
\hline Property & Value \\
\hline Particle Size, $\mu \mathrm{m}$ & $70-150$ \\
\hline $\mathrm{pH}$ & 5.5 \\
\hline Bulk Density, $\mathrm{g} / \mathrm{l}$ & $100-145$ \\
\hline Humidity, $\%$ & $\leq 6$ \\
\hline
\end{tabular}

infinitely adjustable and ranges from 0 to $120 \mathrm{rev} /$ $\mathrm{min}$, while the motor power is $5.5 \mathrm{~kW}$.

The first of the employed mixing elements, the pineapple mixer, is shown in Figure 3. This mixer has a series of rows of rhomboidal diamonds on its surface, spaced by the same distance. This element is used for distributive mixing. Its main function is dividing and recombining the flow passing through the mixer. Due to their deep channels, low pressure drops and stresses are expected [10].

The pineapple mixer has 66 rhomboidal diamonds arranged in 6 equidistant rows on the surface. The distance between the rhomboids in every direction is $6.50 \mathrm{~mm}$. Every rhomboidal diamonds has the same cross-sectional area of

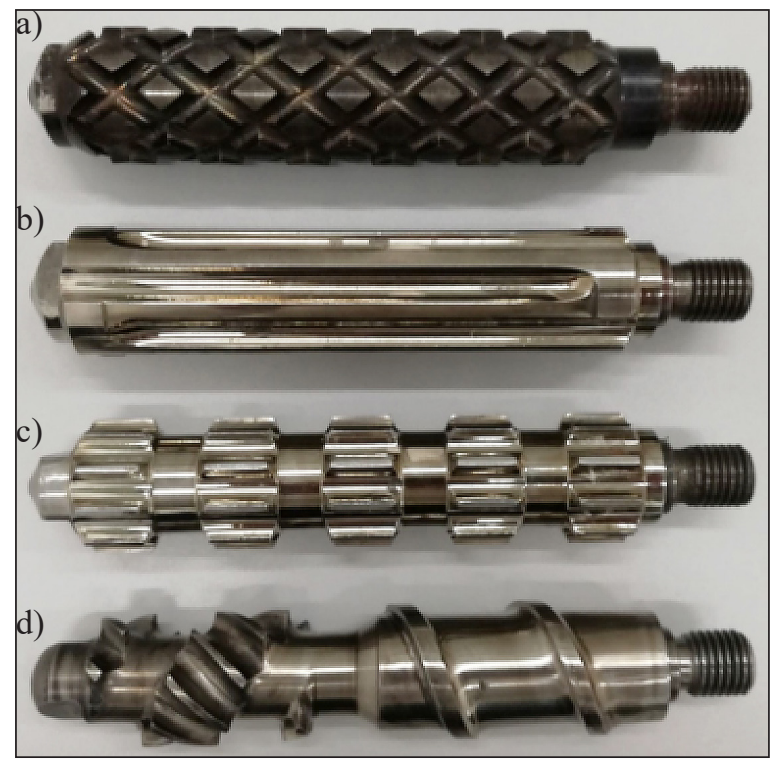

Fig. 2. View of mixing sections for improved shearing and mixing: a) pineapple mixer, b) Maddock mixer, c) cut ring mixer, d) helical mixer 


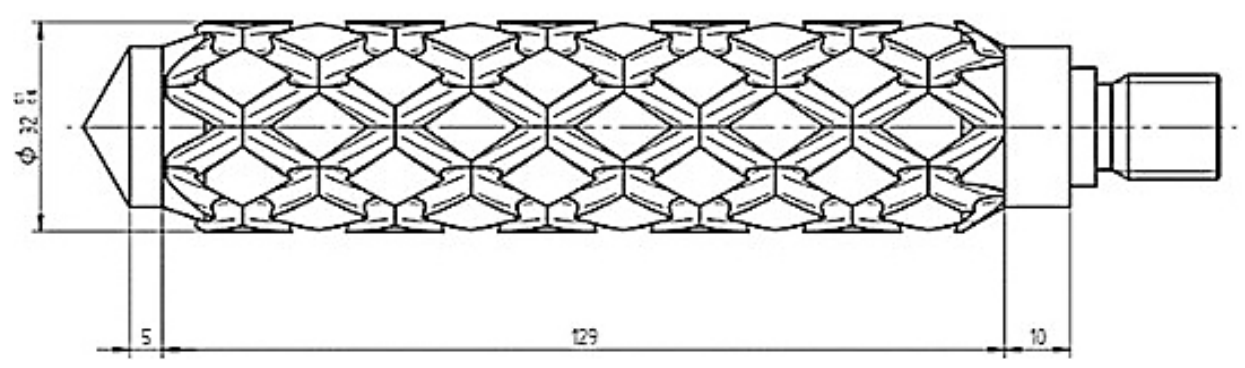

Fig. 3. Pineapple mixer and its dimensions

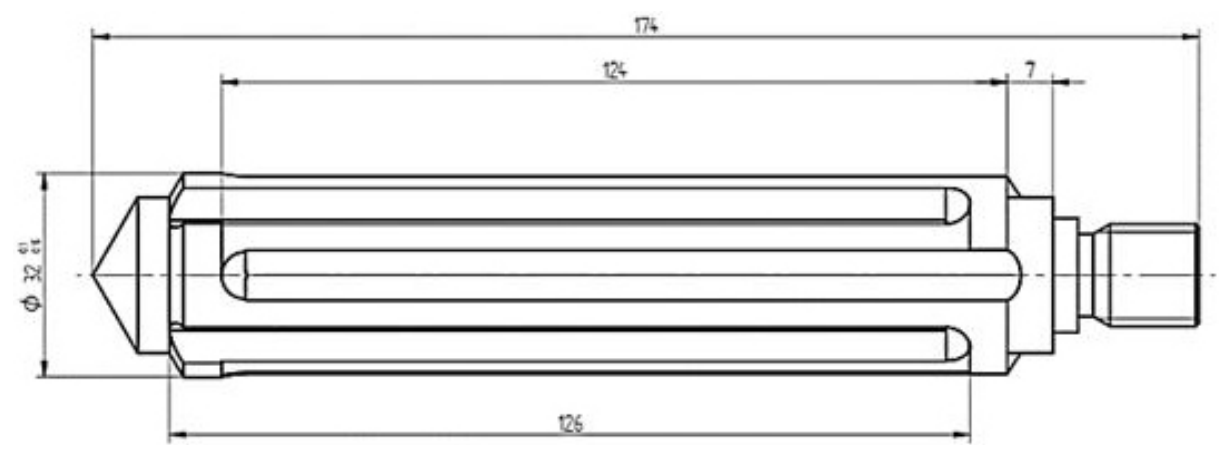

Fig. 4. Maddock mixer and its dimensions

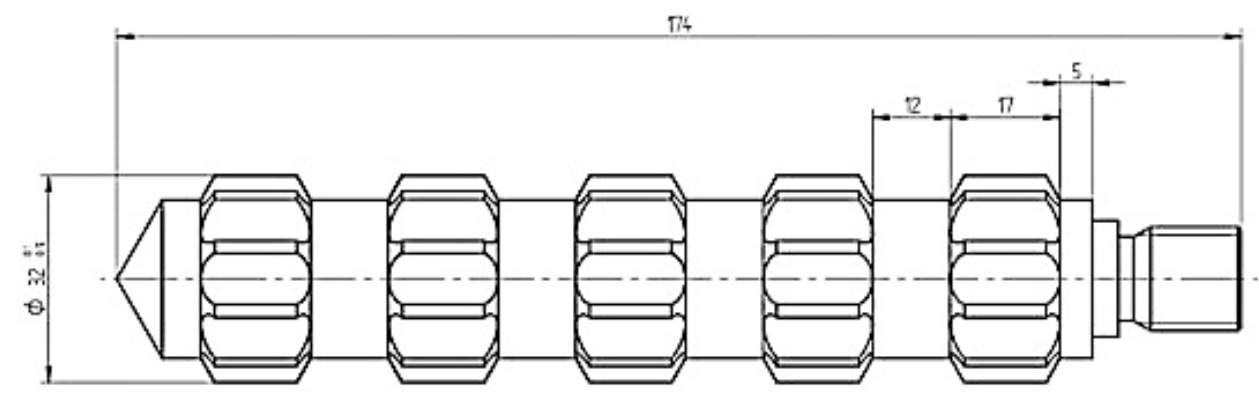

Fig. 5. Cut ring mixer and its dimensions

$6.90 \times 6.90 \mathrm{~mm}$. The channel depth between the rhomboidal diamonds is $3.50 \mathrm{~mm}$.

The Maddock mixer (Fig. 4) is a series of grooves (the melt does not flow between them), one of which is an inlet groove (open at the entry and closed at the exit) and the other is an outlet groove (closed at the entry and open at the exit). The inlet groove is separated from the outlet groove by a barrier flight; the melt flows over the flight and undergoes high shear in the channel between the barrier flight and the extruder barrel [17]. The grooves can be rectilinear or helical with different cross-sectional shapes - the mixer used in this study had rectilinear grooves. Apart from shearing, the Maddock mixer is also used for dispersive mixing.

The Maddock mixer has a grooved structure with 4 inlet and 4 outlet grooves, each being of $125 \mathrm{~mm}$ in length and $3.50 \mathrm{~mm}$ in depth. Between the grooves there is a $4.10 \mathrm{~mm}$ wide barrier flight.
Another mixing section used in the study has five rings, each ring having longitudinal grooves (Fig. 5). This mixer is predominantly used for dispersive mixing. The cut ring mixer has 5 rings of $17 \mathrm{~mm}$ in width, spaced $12 \mathrm{~mm}$ apart. Each ring has 10 teeth and the tooth width is $2 \mathrm{~mm}$. The channel depth between the rings is $3.6 \mathrm{~mm}$.

This mixer consists of two different working parts. One part has a channel, its bottom being the surface of the element, while the side walls are formed by the active and passive surfaces of the helices. The element is bevelled in the centre. The other part of this mixing element has teeth with longitudinal grooves between them. This mixer is used for distributive and dispersive mixing.

The first part of the helical mixer (Fig. 6) consists of three helices with a helix pitch of $31 \mathrm{~mm}$ and a height of $2.35 \mathrm{~mm}$. In the centre, the mixer is a bevelled core of $6.5 \mathrm{~mm} \times 45^{\circ}$. The other part 


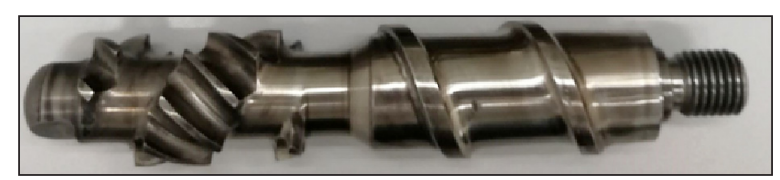

Fig. 6. Helical mixer and its dimensions

of the mixer is made of 15 teeth of various lengths from $4 \mathrm{~mm}$ to $18.5 \mathrm{~mm}$, the helix pitch is $33 \mathrm{~mm}$, the tooth width is $2 \mathrm{~mm}$, and the depth between the teeth is $5.5 \mathrm{~mm}$.

A strip die is attached to the plasticizing system of the T32-25 extruder. This flat sheet die has the dimensions of $20.19 \mathrm{~mm} \times 1.99 \mathrm{~mm}$. The experiments were performed without the use of a calibration device.

The test stand was equipped with instruments and devices enabling measurement of the tested parameters, such as power supplied to the extruder, temperature of the extrudate, screw rotation speed, as well as length, weight and extrusion time of the extrudate.

\section{Research program and methods}

To identify the effect of intensive mixing and shearing sections on the extrusion of wood polymer composites, we designed a research programme considering the filler content, temperatures in the plasticizing system and extruder die as well as the screw rotation speed.

Before starting the T32-25 extruder, the polypropylene was mixed with the filler in a ratio of $5,10,15$ and $20 \mathrm{wt} \%$, respectively. A given filler content was added to $1000 \mathrm{~g}$ of the polymeric material. The materials were mixed together and the mixture was fed into the hopper.

Before the extrusion process, it was necessary to set the temperatures of individual heaters in the plasticizing system. Based on the values reported in the literature and our previous experience, these temperatures were set equal to 160,180 , 190 and $195^{\circ} \mathrm{C}$, respectively; the temperature of the extrusion dies was $190^{\circ} \mathrm{C}$. The tests were carried out with one screw rotation speed of $45 \mathrm{rpm}$. Every time the extrusion conditions were modified, no measurements were made until the process became stable, which occurred after about 30 minutes. The extrusion process was continued until the plasticizing system was completely empty. With the plasticizing system becoming empty, the mixing and shearing elements were changed, and another portion of the prepared mixture with different filler content was fed into the hopper.
The research programme involved measuring the power supplied to the extruder, temperature of the extrudate, screw rotation speed, extrusion time, weight and geometry of the extrudate. The above calculations were followed by measurement of the mass flow rate of the extrudate leaving the extrusion die, this parameter being a measure of extrusion process efficiency. Unit energy consumption was determined based on measurements of the power supplied to the extruder and mass flow rate. Another stage of the study involved calculating the extrusion speed and energy efficiency of the extrusion process of filled polypropylene samples. Measurements of the melt flow rate of wood fibre-filled polypropylene were made using the Zwick 4105.100 plastometer (Zwick Roell, Ulm, Germany) equipped with additional instruments and analytical balance PRL TA14. Melt flow rate measurements were made in compliance with the ISO 1133 standard.

The Barus effect was investigated in order to determine the swell of the extrudate stream leaving the die caused by differences in normal stresses during melt flow. Barus effect measurements were made after cooling the extrudate at normal temperature. The numerical value of this effect was determined using the ratio between the extrudate cross-sectional area and the cross-sectional area of the extrusion die, expressed as percentage.

\section{RESULTS AND DISCUSSION}

Results were used to establish relationships between the extrusion process parameters, such as power supplied to the extruder, extrudate temperature, screw rotation speed, extrusion speed, mass flow rate, unit energy consumption, mass flow rate and Barus effect, as well as natural filler content and employed intensive mixing and shearing elements.

\section{Power supplied to the extruder}

Figure 7 shows the variations in the power supplied to the extruder in the extrusion process for polypropylene samples containing different filler contents, conducted with the use of the analysed mixing sections. Regardless of the filler content and mixer used, the power supplied to the machine decreases, which correlates with the behaviour pattern of the screw rotation speed plotted in Figure 9. For the filler content 


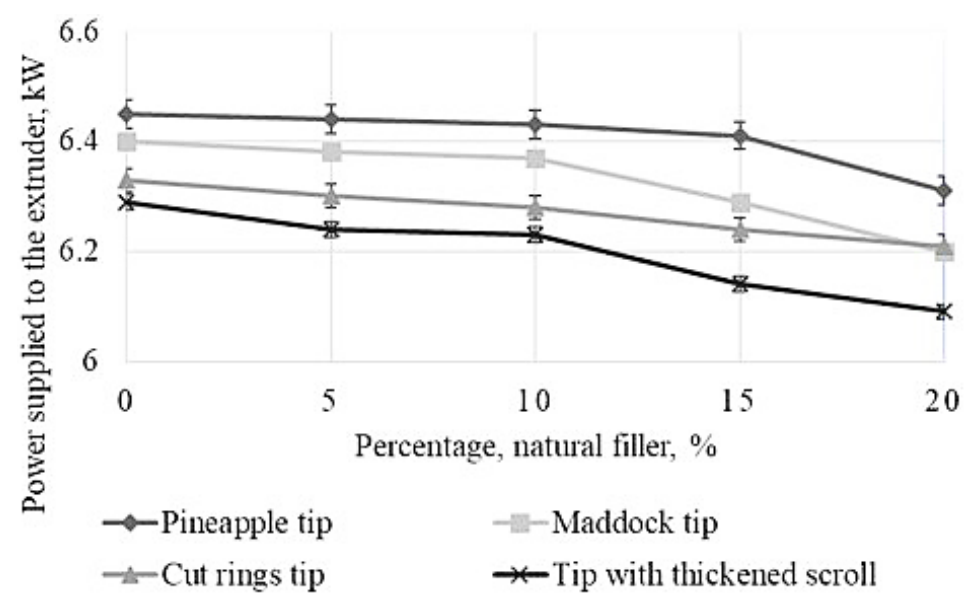

Fig. 7. Power supplied to the extruder versus natural filler content

ranging from 0 to $10 \%$ the decrease in the power is small, but it becomes more significant with increasing the filler content.

The highest decrease in the power supplied to the extruder for the tested filler contents is observed for the extrusion process conducted with the Maddock mixer and helical mixer and it is equal $0.2 \mathrm{~kW}$, which amounts to approximately $3.15 \%$ of the initial value obtained with this mixer for the unfilled sample. The reduction in power is $0.14 \%$ for the pineapple mixer and $0.12 \%$ for the cut ring mixer. The power supplied to the extruder decreases with the increase of the filler content, which may be due to the increased slip at the wall, which in turn reduced the resistance to the material flow, resulting in the observed increase in the extrusion speed and, consequently, a slight increase in the mass flow rate, which is a favorable and desirable phenomenon [18].

\section{Extrudate temperature}

Figure 8 shows the temperature of the extrudate filled with different filler contents, extruded with the use of four different mixing and shearing elements. Irrespective of the element used, the addition of $5 \mathrm{wt} \%$ of the filler causes a decrease in the extrudate temperature. The highest decrease in the extrudate temperature can be observed when the extrusion process is conducted using the Maddock mixer - the temperature drops by $3.4 \%$, which amounts to a decrease by $7{ }^{\circ} \mathrm{C}$; the lowest decrease in the extrudate temperature is observed when the helical mixer is used - the temperature drops by $1.02 \%$, which amounts to a $2{ }^{\circ} \mathrm{C}$ decrease compared to the value obtained for the unfilled polymer sample. The addition of a higher filler content causes a slight increase in the extrudate temperature at the beginning; then, the temperature becomes stable at a lower level than that of the unfilled polypropylene. This trend can be observed in the extrusion process conducted with the 4 tested mixing and shearing elements. By analysing temperature variations in the extrusion of different plastics, one can evaluate to a certain extent the thermal stability of the process, and hence maintain homogenous physical structure and properties of the extrudate during the extrusion process. The results demonstrate that the addition of the filler to the propylene and the use of different mixing and shearing elements affect thermal phenomena occurring in the conventional extrusion process. The observed increase in the temperature of the extrudate containing $10-20 \mathrm{wt} \%$ of the filler may result from the characteristics of the filler itself. A slight increase in the screw rotation speed (Fig. 9) and filler content generates heat due to friction of filler particles against the working surface of the barrel walls, screw and screw mixers, which, consequently, leads to an increase in the temperature of the extrudate. The highest increase in the temperature of the polypropylene sample containing $10-20 \mathrm{wt} \%$ of the natural filler and extruded with the use of the Maddock mixer may result from the fact that the melt flow is predominantly rotational here, while the other key direction of material flow is that along the axis of the mixer. The combination of these two flows probably causes the melt to pass at least once through the inlet and outlet grooves and barrier flight [19]. The occurrence of the two types of flow probably results in intensive generation of friction heat. 


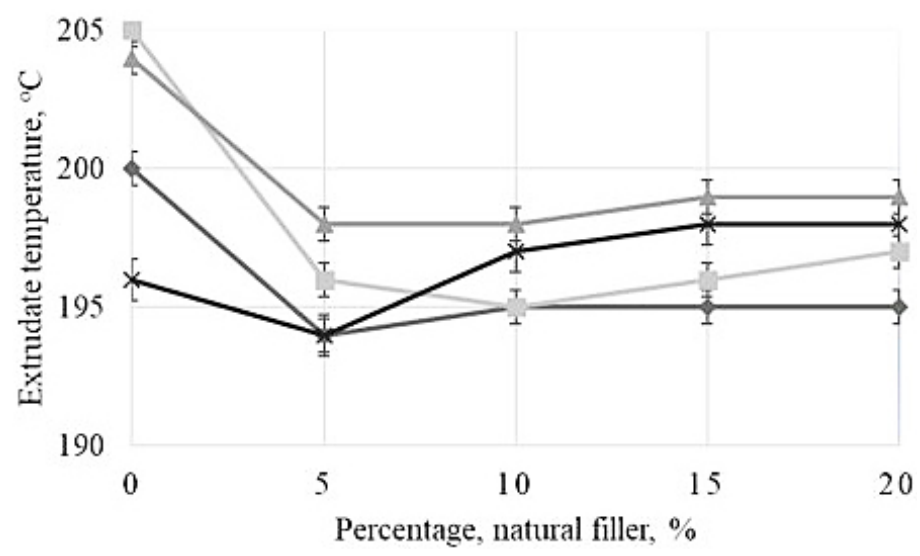

Fig. 8. Extrudate temperature versus natural filler content; the denotations are the same as in Figure 7

\section{Screw rotation speed}

The effect of the natural filler (wood fibre) content on the screw rotation speed in the analysed extrusion process conducted with the use of different mixing and shearing elements is plotted in Figure 9. An analysis of the data in the figure reveals that the screw rotation speed slightly increases with increasing the filler content. It can be observed that the screw rotation speed increases with the use of every tested mixing and shearing element. On increasing the filler content from 0 to $20 \%$ and using the pineapple mixer, the screw rotation speed increases on average by $1.9 \mathrm{rpm}$, which amounts to a $4.22 \%$ increase compared to the unfilled plastic.

In the case of the Maddock mixer and helical mixer, the screw rotation speed increases on average by $1.2 \mathrm{rpm}$, which amounts to an increase by $2.63 \%$ and $2.59 \%$, respectively. When the cut ring mixer is used, the screw rotation speed increase is very small and amounts to $0.6 \mathrm{rpm}$, which amounts to an increase by $1.29 \%$. The observed variations in screw rotation speed undoubtedly result from both the geometry of the employed mixing and shearing elements and the increased natural filler content, thus leading to the change in rheological properties of the processed polymeric composition. The addition of wood fibre may have a significant effect on the rheological properties of WPCs [16]. The small increase in the screw rotation speed probably results from the fact that pinewood contains a significant amount of fatty acid that may migrate to the surface and act as an external lubricant [20].

It is also worth focusing on the effect of temperature. Although the natural filler is in solid state, the viscosity of WPC melt is significantly reduced at elevated temperatures due to increased motion of polymer chains [21]. The slight increase in the screw rotation speed leads to an increase in the mass flow rate (Fig. 11) and extrusion speed (Fig. 10). The highest increase in the screw rotation speed is obtained with the pineapple mixer. This mixing element is predominantly used for distributive mixing to ensure even distribution of the filler over the entire polymeric matrix. In this mixer the rotational flow is combined with splitting motion ahead of each rhomboidal diamond.

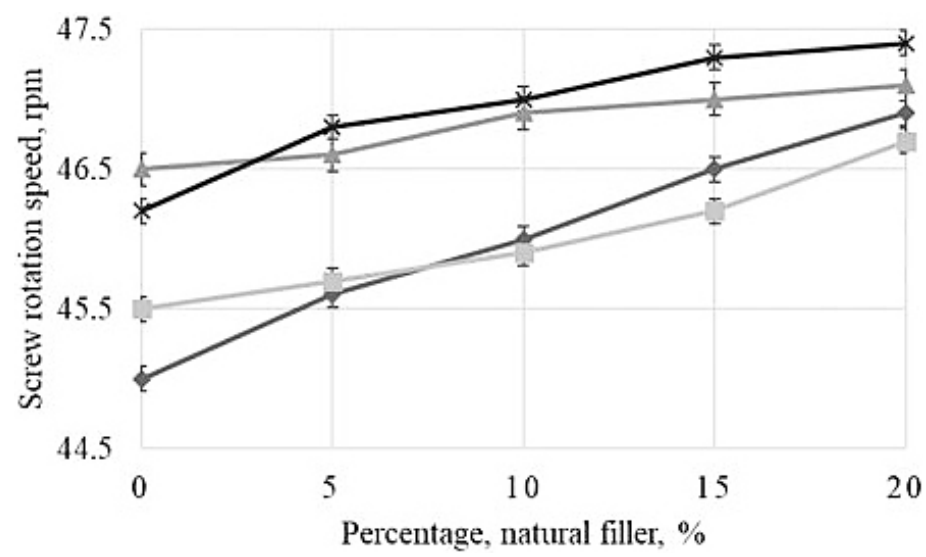

Fig. 9. Screw rotation speed versus natural filler content; the denotations are the same as in Figure 7 


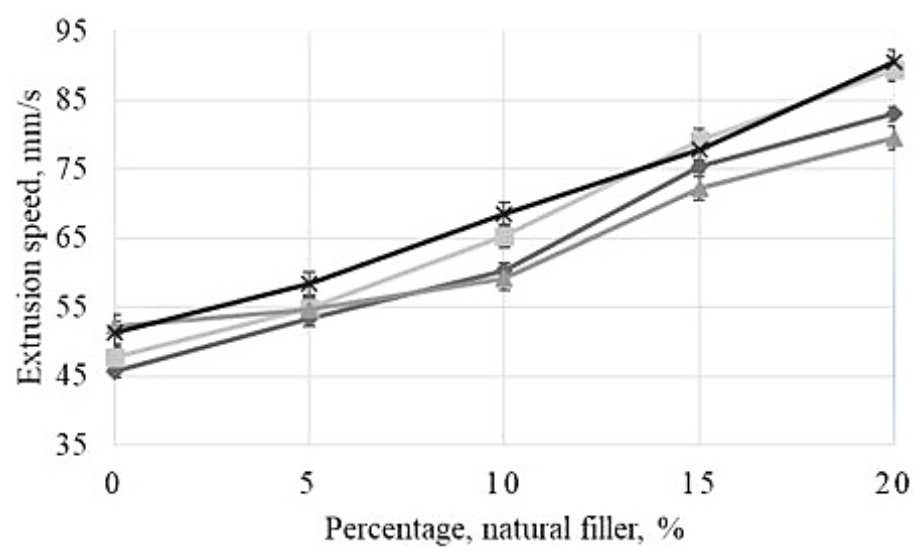

Fig. 10. Extrusion speed versus natural filler content, the denotations are the same as in Figure 7

The highest flow rate can be observed in the vicinity of the rhomboidal diamond. Also, the flow rate is high in the gap between the rhomboidal diamond and the barrel [19], which can be explained by a slight increase in the screw rotation speed.

\section{Extrusion speed}

Considering the tested filler contents, the highest increase in the extrusion speed is obtained when the extrusion process is conducted with the use of the Maddock mixer (Fig. 10). With this mixer, the extrusion speed is $47.66 \mathrm{~mm} / \mathrm{s}$ for the unfilled polymer sample and $89.41 \mathrm{~mm} / \mathrm{s}$ for the sample containing $20 \mathrm{wt} \%$ of the filler, which amounts to an $87.6 \%$ increase compared to the value obtained for the unfilled sample. This can be explained by the fact that increasing the filler content leads to an increase in the sliding speed of the polymer; as shown in studies [22-26], sliding properties are significantly affected by the presence of wood fibre. This effect can probably be observed in this study, hence the increase in the extrusion speed. This explanation seems correct, considering the mass flow rate increase (Fig. 11) and MFR behaviour pattern (Fig. 13). The highest difference in extrusion speeds obtained with different mixing and shearing elements can be observed for the polymer sample containing $10 \mathrm{wt} \%$ of the filler. The extrusion speed obtained with the helical mixer is $68.47 \mathrm{~mm} / \mathrm{s}$, which is $16 \%$ higher than the value obtained with the cut ring mixer, i.e. $59.1 \mathrm{~mm} / \mathrm{s}$. In addition, when extruding a tape-shaped product through a slit die head, the velocity profile of the flowing out polymer on the cross section will be variable, leading to the observed slight flow instability [27].

\section{Mass flow rate of the extrudate}

Figure 11 shows the results of mass flow rate depending on the filler content and employed mixing and shearing elements. It can be observed that the mass flow fate increases with the filler content, irrespective of the element type used. This can be attributed to the fact that the density of the polymeric material increases with the filler content, which results from a higher

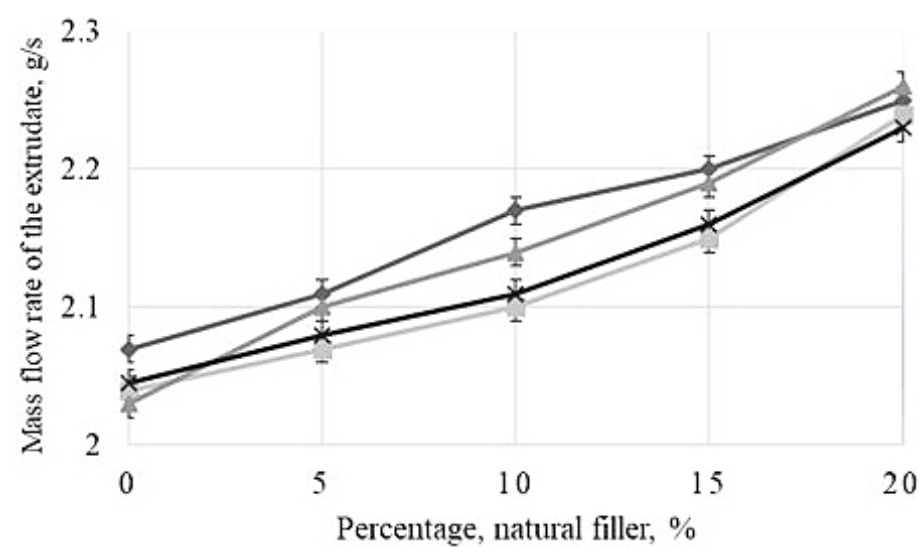

Fig. 11. Mass flow rate versus natural filler content; the denotations are the same as in Figure 7 


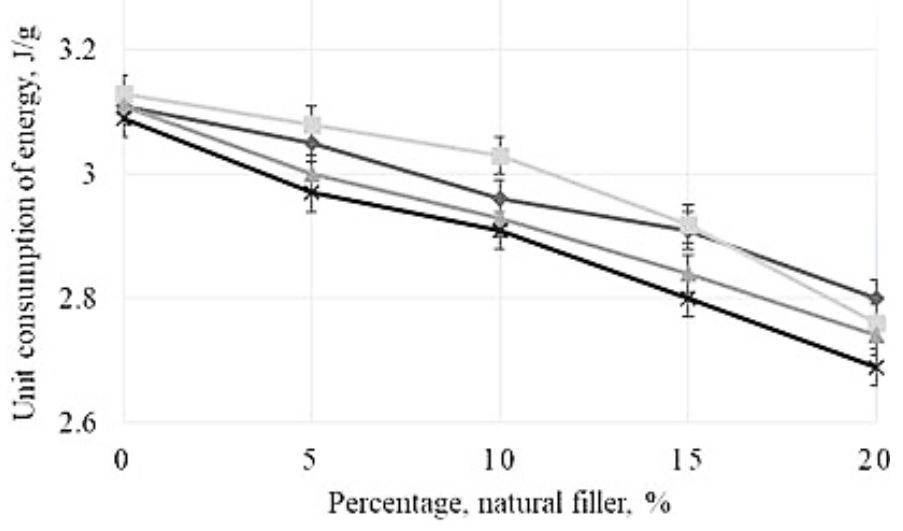

Fig. 12. Unit consumption of energy versus natural filler content; the denotations are the same as in Figure 7

density of wood fibre compared to the density of PP. Among all tested mixing and shearing elements, the highest mass flow rate is obtained with the pineapple mixer for $0 \mathrm{wt} \%, 5 \mathrm{wt} \%, 10$ $\mathrm{wt} \%$ and $15 \mathrm{wt} \%$ of the filler. The lowest mass flow rate values are obtained with the use of the Maddock mixer for $5 \mathrm{wt} \%, 10 \mathrm{wt} \%$ and 15 wt $\%$ of the filler. The differences in the obtained mass flow rates are the most significant for the polymer samples containing $10 \mathrm{wt} \%$ of the filler. The mass flow rate obtained with the pineapple mixer is $2.17 \mathrm{~g} / \mathrm{s}$, while that obtained with the Maddock mixer is $2.1 \mathrm{~g} / \mathrm{s}$, which amounts to a $3.3 \%$ decrease in the mass flow rate compared to that obtained with the pineapple mixer.

It results from the fact that the volume of the flow channels in the Maddock mixer is lower and thus the melt flow resistance of this mixer is higher than that of the cut ring mixer in which the flow channel volume is higher and thus the melt flow is more efficient. In addition, it should be stressed that in the Maddock mixer the flow is predominantly radial, while in the pineapple mixer it is axial flow that is dominant [19]. For the tested filler contents, the highest increase in mass flow rate can be observed when the cut ring mixer is used. The mass flow rate obtained with this mixer for the unfilled polymer sample is $2.03 \mathrm{~g} / \mathrm{s}$ and $2.26 \mathrm{~g} / \mathrm{s}$ for the sample containing $20 \mathrm{wt} \%$ filler, which amounts to an $11.3 \%$ increase compared to the unfilled polymer sample.

\section{Unite consumption of energy}

The relationship between the unit consumption of energy and the filler content is plotted in Figure 12. It can be observed that the unit consumption of energy decreases with increasing the filler content, which is confirmed by the results of power supplied to the extruder (Fig. 7) and mass flow rate (Fig. 11). When the power supplied to the extruder is reduced and the mass flow rate is increased, the unit consumption of energy decreases, which is a well-known phenomenon often described in the literature [28]. For the tested filler contents, the highest unit energy consumption of $0.4 \mathrm{~J} / \mathrm{g}$ is obtained when the helical mixer is used, which is almost $13 \%$ of the initial value. As for other mixing and shearing elements, the variation in the unit energy consumption is also significant and ranges from 0.33 to $0.4 \mathrm{~J} / \mathrm{g}$, which is, relatively, between $10.6 \%$ and $12 \%$ of the value obtained for the unfilled polymer sample.

\section{Melt flow rate}

MFR measurements were made in compliance with the EN ISO 1133-1:2011standard. As expected, the MFR decreases with increasing the filler content. Figure 13 illustrates the relationship between the decrease in MFR with increasing the filler content. The highest decrease in the MFR for the tested filler contents can be observed when the helical mixer is used. For this mixer, the MFR is $6.97 \mathrm{~g} / 10 \mathrm{~min}$ for the unfilled polymer and $1.63 \mathrm{~g} / 10 \mathrm{~min}$ for the polymer sample containing $20 \mathrm{wt} \%$ filler, which amounts to $76.6 \%$ of the initial value. The decrease in MFR with increasing the filler content results from an increased viscosity of the filled polymer and thus its higher density due to increased content of wood fibre particles in the propylene matrix. This phenomenon is well known and often described in the literature [22-26, 29-30]. The greatest difference between the MFR values obtained with different mixers can be observed for the polymer sample containing $15 \mathrm{wt} \%$ filler. For this filler content, the MFR obtained with the cut ring mixer is $1.98 \mathrm{~g} / 10 \mathrm{~min}$, 


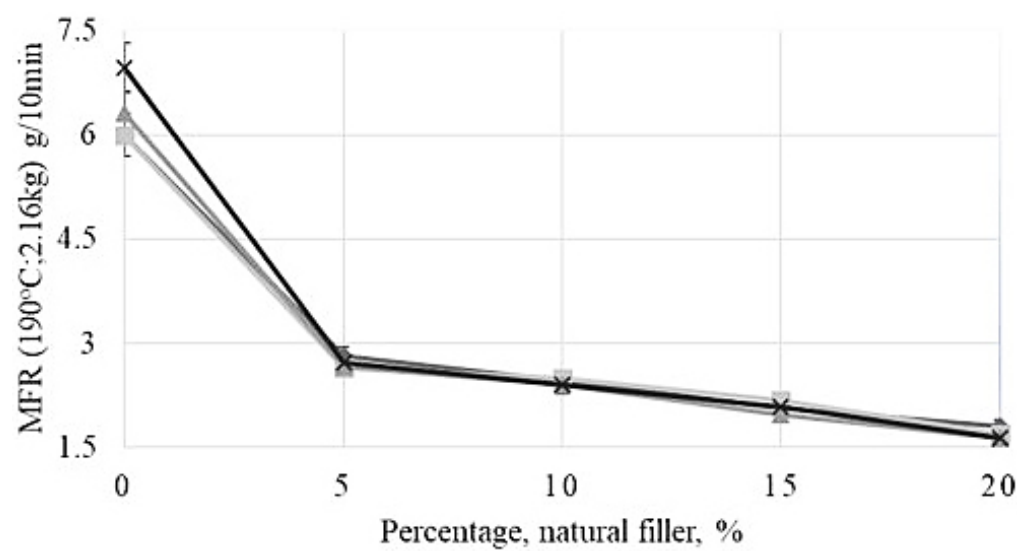

Fig. 13. Melt flow rate versus natural filler content; the denotations are the same as in Figure 7

which is $9 \%$ lower than the MFR value obtained with the Maddock mixer (2.18 g/10min). This difference probably results from that fact that the cut ring mixer is primarily distributive, with filler particles better distributed in the matrix, which means higher viscosity and decreased MFR when compared to the Maddock mixer where dispersive characteristics are dominant.

\section{Barus effect}

Figure 14 illustrates the effect of the tested intensive mixing and shearing elements on the Barus effect in relation to the natural filler content. It can be observed that the filler content in the polymer and the type of mixing and shearing element used are of significant importance. The addition of 20 $\mathrm{wt} \%$ of the filler to the polypropylene matrix causes a significant reduction in the Barus effect. The reduction in the swell of the stream of the polymeric material, in relation to the unfilled material, ranges between 15.5 and 19.5\%. Moreover, the highest decrease in the numerical value of the extrudate swell is obtained with the Maddock mixer, this value being $18.38 \%$ compared to the unfilled material, and the lowest decrease of $15.66 \%$ is obtained with the cut ring mixer. Regarding the pineapple and helical mixers, the reduction in the Barus effect is similar and amounts, respectively, to $16.46 \%$ and $17.73 \%$ when compared to pure PP. The die swell of the polymeric material stream is reduced after the introduction of the filler into the polymeric matrix. This can be explained by stiffening of the polymeric material and thus its limited elastic recovery, which is the main cause of die swell.

The beneficial effect of wood fibre content on the Barus effect is very important from the point of view of polymer processing. The Maddock mixer, which ensures the highest decrease in the numerical value of the Barus effect for the tested composites, is one of the elements used for dispersive mixing of filler particles by inducing high shear forces. The decreased Barus effect for the tested materials is probably caused by high shear forces that mechanically reduce the size of filler particles introduced into the polymeric matrix.

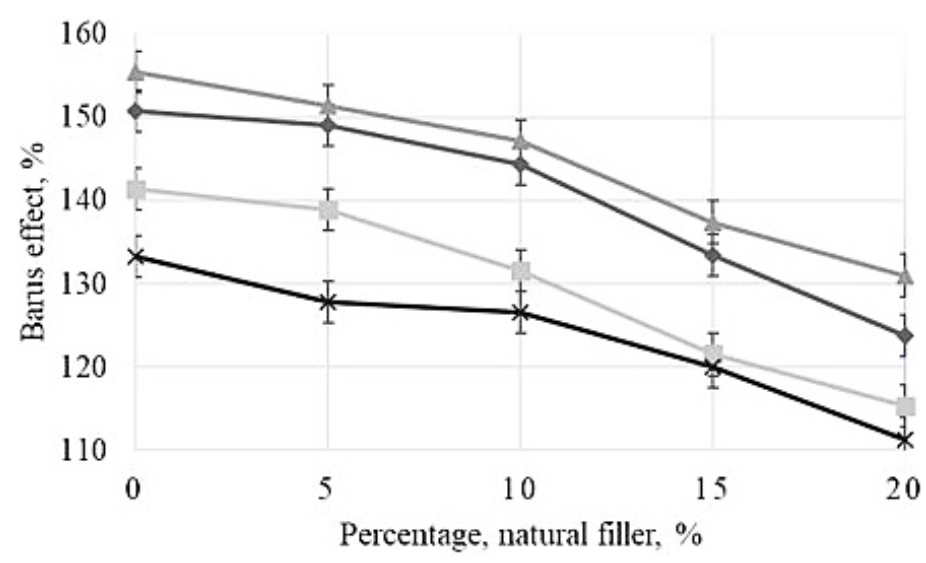

Fig. 14. Barus effect versus natural filler content; the denotations are the same as in Figure 7 


\section{CONCLUSIONS}

Single-screw extrusion is one of the most important polymer processing methods. The screw and barrel are the critical components of the plasticizing system and significantly affects the extrusion process. The efficiency and quality of plasticization of the polymeric material, including its structure homogenization, depend on the screw and barrel design. The objective of the study described in this paper was to determine the effect of using four different intensive mixing and shearing elements of the screw on the effectiveness of the extrusion process for polypropylene filled with different wood fibre contents.

The mixing element acts as a choke, lowering the efficiency, but allows the process to be carried out at a higher rotational speed of the screw and homogenizes the viscosity of the plasticized material leaving the shearing element while ensuring simultaneous tension relaxation in the material. The quality of the mixing element, i.e. the effectiveness of its operation, is determined by e.g. equalization of the temperature of the material at the end of the screw. The shearing elements accelerate the uniform plasticization of the material by intensifying the transformation of the mechanical energy of the screw drive into thermal energy, obtained as a result of the high shear rate of the plasticized material. The use of the mixing and shearing element increases the power consumed by the drive, i.e. at the same time the temperature of the plasticized material increases. The temperature increase of the material flowing through the gap depends on whether it is less or more warm since the temperature of the colder material (with higher viscosity) will increase faster than the more heated material (with lower viscosity).

The results clearly demonstrate that the addition of different contents of the natural filler (wood fibre) to polypropylene as well as the use of the tested intensive mixing and shearing elements have a positive effect on the extrusion process, and that the effectiveness of this process increases with the filler content. It has been shown that rheological phenomena occurring during melt flow through the plasticizing system, and thus the efficiency of the extrusion process can be improved by using different intensive mixing and shearing elements, without modifying the extrusion process parameters, such as screw rotation speed and temperature.

\section{Acknowledgments}

The project/research was financed in the framework of the project Lublin University of Technology-Regional Excellence Initiative, funded by the Polish Ministry of Science and Higher Education (contract no. 030/RID/2018/19).

\section{REFERENCES}

1. Jung H., White J.L. Investigation of melting phenomena in modular co-rotating twin screw extrusion. International Polymer Processing. 2003; 18: 127-132.

2. Rosato D.V. Extruding Plastics: A Practical Processing Handbook. Springer Science \& Business Media. 2013; 208-213.

3. Głogowska K., Sikora J., Dulebova L. Properties of Moldings Prepared from LDPE-Pumpkin Seed Hulls Blend. Advances in Science and Technology Research Journal. 2017; 11(4): 318-325.

4. Wilczyński K., Nastaj A., Lewandowski A., Wilczyński K.J., Buziak K. Fundamentals of Global Modeling for Polymer Extrusion. Polymer. 2019; 11: 2106.

5. Mount E.M. 12 - Extrusion Processes. In Applied Plastics Engineering Handbook: Processing, Materials, and Applications (Second Edition); Kutz, M., Ed.; Plastics Design Library; William Andrew Publishing; 2016.

6. Sikora J.W. Comparison between LDPE conventional and autothermal extrusion characteristics. International Polymer Processing. 1998; 13: 9-12.

7. Jian R., Yang W., Cheng L., Xie P. Numerical analysis of enhanced heat transfer by incorporating torsion elements in the homogenizing section of polymer plasticization with the field synergy principle. International Journal of Heat and Mass Transfer - Journals. 2017; 15; 946-953.

8. Kelly A.L., Brown E.C., Coates P.D. The effect of screw geometry on melt temperature profile in single screw extrusion. Polymer Engineering and Science. 2006; 46: 1706-1714.

9. Bur A.J., Roth S.C., Spalding M.A., Baugh, D.W., Koppi K.A., Buzanowski W.C. Temperature gradients in the channels of a single-screw extruder. Polymer Engineering and Science. 2004; 44: 2148-2157.

10. Chung C.I. Mixing study on different pineapple mixer designs-simulation results. Proceedings of SPE ANTEC, Detroit, United States, May 2000, Technomic Publishing Co, U.S.; 2000.

11. Rauwendaal C. Polymer Extrusion. Carl Hanser Verlag: Munich, Germany; 2014.

12. Rauwendaal C. Polymer mixing: A self-study guide. Carl Hanser Verlag: Munich, Germany; 1998. 
13. Zitzenbacher G., Karlbauer R., Thiel H. A new calculation model and optimization method for maddock mixers in single screw plasticising technology. International Polymer Processing. 2007; 22: 73-8.

14. Sikora J.W. Increasing the efficiency of the extrusion process. Polymer Engineering and Science. 2008; 48: 1678-1682.

15. Głogowska K., Sikora J., Majewski Ł. New concepts for the active grooved-feed section extruder. International Journal of Industrial Engineering and Management 4.0. 2017; 5: 237-240.

16. Mazzanti V., Mollica F. A Review of Wood Polymer Composites Rheology and Its Implications for Processing. Polymer. 2020; 12: 2304.

17. Potente H., Többen W.H. Improved design of shearing sections with new calculation models based on 3D finite-element simulations. Macromolecular Materials and Engineering. 2020; 287: 808-814.

18. Sikora J.W., Kapusniak T. Efficiency of the extrusion process and characteristics of a conical-circular die. Polimery. 2005; 50: 748-754.

19. Hopmann C., Schön M., Reul M.M., Facklam M. A Method for the Validation of Simulated Mixing Characteristics of Two Dynamic Mixers in SingleScrew Extrusion. Polymer. 2020; 12: 2234.

20. Li T.Q., Wolcott M.P. Rheology of wood plastics melt. Part 1. Capillary rheometry of HDPE filled with maple. Polymer Engineering Science. 2005; 45: 549-559.

21. Kaseem M., Hamad K., Deri F., Ko Y.G. Effect of wood fibers on the rheological and mechanical properties of polystyrene/wood composites. Journal of Wood Chemistry and Technology. 2017; 37: 251-260.

22. Duretek I., Schuschnigg S., Gooneie A., Langecker G.R., Holzer C. Rheological properties of wood poly- mer composites and their role in extrusion. Journal of Physics: Conference Series. 2015; 602: 012014.

23. Li T.Q., Wolcott M.P. Rheology of HDPE-wood composites. I. Steady state shear and extensional flow. Composites Part A. 2004: 35; 303-311.

24. Carrino L., Ciliberto S., Giorleo G., Prisco U. Effect of filler content and temperature on steady-state shear flow of wood/high density polyethylene composites. Polymer Composites. 2011; 32: 796-809.

25. Hristov V., Takacs E., Vlachopoulos J. Surface tearing and wall slip phenomena in extrusion of highly filled HDPE/wood flour composites. Polymer Engineering Science. 2006; 46: 1204-1214.

26. Hristov V., Vlachopoulos J. Thermoplastic silicone elastomer lubricant in extrusion of polypropylene wood flour composites. Advances Polymer Technology. 2007; 26: 100-108.

27. Barczewski M., Barczewski R., Chwalczuk T. The in-line detection method of sharkskin melt flow instability during polyethylene extrusion based on pressure analysis. Journal of Manufacturing Processes. 2020; 59: 53-166.

28. Sasimowski E., Sikora J.W. Królikowski, B. Effectiveness of Polyethylene Extrusion in a Single-Screw Grooved Feed Extruder. Polimery. 2014; 59: 505-510.

29. Li H., Law S., Sain M. Process rheology and mechanical property correlation ship of wood floorpolypropylene composites. Journal of Reinforced Plastics and Composites. 2004; 23: 1153-1158.

30. Maiti S.N., Subbarao, R., Ibrahim M.N. Effect of wood fibers on the rheological properties of i-PP/ wood fiber composites. Journal of Applied Polymer Science. 2004; 91: 644-650. 